\title{
Treatment of Periprosthetic Femoral Fractures Following Hip Arthroplasty
}

\author{
Joong-Myung Lee, MD, PhD, Tae-sup Kim, MD, Tae-ho Kim, MD \\ Department of Orthopaedic Surgery, CHA Bundang Medical Center, CHA University, Seongnam, Korea
}

Purpose: This study was performed to assess potential improvements in clinical outcomes when applying recent advanced hip arthroplasty surgical techniques and understand the potential relationship between bone mineral density (BMD) and surgical outcomes.

Materials and Methods: Among 37 cases of periprosthetic femoral fractures after hip arthroplasty treated between March 2014 and September 2016, all included a follow-up of at least one year and were included in this study. Outcomes were evaluated using the Beals and Tower's criteria. BMD was examined in 27 of 37 cases and the relationship between osteoporosis and treatment outcomes was analyzed. Advanced hip arthroplasty surgical approaches varied depending on the fracture type: i) open reduction with wiring for Vancouver A, ii) open reduction with double plate fixation for Vancouver B1, iii) revision THA with long stem for Vancouver B1Nonunion, B2 and B3, and iv) open reduction with double plate fixation for Vancouver C.

Results: When assessed using the Beals and Tower's criteria, 33 out of $37(89.2 \%)$ patients were excellent and 4 $(10.8 \%)$ were poor. These outcomes were an improvement compared with series I (81.8\%). When analyzed according to the Vancouver classification, patients with type A $(n=8)$, type B1 $(n=16)$, and type B2 $(n=2)$ were all excellent, the patients with type B3 were excellent $(n=1)$ and poor $(n=1)$, and the patients with type $C$ were excellent $(n=6)$ and poor $(n=3)$. The mean BMD was -2.6 (T-score) in 27 of 37 cases and -4.4 in 4 cases with poor prognosis. Osteoporosis was statistically correlated to those classified as poor by Beals and Tower.

Conclusion: The results of the analysis suggest that applying new surgical hip arthroplasty treatment approaches leads to improved outcomes compared with the author's previous study. When treating periprosthetic femoral fractures following total hip arthroplasty, an appropriate internal fixation method should be selected, at least in part based on the Vancouver classification. In addition, osteoporosis may be a major prognostic factor for the outcomes of surgical treatment.

Key Words: Periprosthetic fracture, Vancouver classification, Hip replacement arthroplasty, Osteoporosis

Submitted: October 14, 2017 1st revision: January 6, 2018

2nd revision: January 20, 2018 Final acceptance: January 23, 2018

Address reprint request to

Joong-Myung Lee, MD, PhD

(https://orcid.org/0000-0001-6241-9534)

Department of Orthopaedic Surgery, CHA Bundang Medical

Center, CHA University, 59 Yatap-ro, Bundang-gu, Seongnam

13496, Korea

TEL: +82-31-780-5289 FAX: +82-31-708-3578

E-mail: drjmleednaver.com
This is an Open Access article distributed under the terms of the Creative Commons Attribution Non-Commercial License (http://creativecommons. org/licenses/by-nc/4.0) which permits unrestricted non-commercial use, distribution, and reproduction in any medium, provided the original work is properly cited. 


\section{INTRODUCTION}

The prevalence of periprosthetic femoral fractures after total hip arthroplasty (THA) continues to rise with an aging populations and ranges between $1.7 \%$ and $3.8 \%{ }^{11}$. Periprosthetic femoral fracture is one common causes of revision THA after osteolysis and recurrent dislocation. Periprosthetic femoral fracture is most frequently caused by minor trauma such as a fall, but does occur, although rarely, in the absence of trauma, implying that this condition may be associated with poor bone quality making it susceptible to fractures ${ }^{2-4}$. Rigid fixation for periprosthetic femoral fractures with screws and plates is challenging due to interference of a preexisting femoral stem, osteoporotic bone quality and others, and fracture healing is difficult to achieve because of cortex thinning because of stress shielding, osteolysis and others ${ }^{5}$. For the treatment of periprosthetic femoral fractures after hip arthroplasty, many authors apply clinical guidelines according to the Vancouver classification. In 2010, the authors of this study achieved excellent or good radiological results in $84.4 \%$ of patients using Beals and Tower's criteria by reviewing the clinical outcomes of 32 cases with periprosthetic femoral fracture according to the management algorithm of the Vancouver classification ${ }^{6}$. In a previous study by these authors, clinical results were poor in Vancouver type B1 and $\mathrm{C}$ fractures, and we concluded that; i) rigid fixation using double plates instead of single-plate fixation is warranted for type $\mathrm{B} 1$ and $\mathrm{C}$ fractures where internal fixation is challenging and ii) the insertion of bone cement may improve the prognosis of Vancouver type $\mathrm{C}$ fractures associated with osteoporosis ${ }^{6}$. In the past, stem loosening was the most common risk factor for periprosthetic femoral fractures with the use of cemented stems in THA. However, a recent trend toward increased use of uncemented stems has decreased the rate of stem loosening, and instead the rate of systemic diseases such as osteoporosis has risen along with the increased incidence of periprosthetic femoral fracture with age $^{7)}$. In response to such changes and the results of author's previous study, modification of clinical practice guidelines for Vancouver fracture types is warranted. This study aims to analyze the surgical results of the new treatment principles and to explore the effect of osteoporosis on clinical outcomes.

\section{MATERIALS AND METHODS}

This study was performed following an approval from the institutional review board and involved a total of 37 patients (37 hips) who received treatment for a periprosthetic femoral fracture after THA from March 2014 to September 2016 with a minimum follow-up of one year. Periprosthetic femoral fractures were classified according to the Vancouver system $^{8,9)}$, and the mean onset period of a fracture following THA was 7 years 3 months (range, 15 days-30 years). The mean age of patients at the time of fracture was 75 years (range, 43-90 years). Sixteen patients were male and 21 were female. The average follow-up period was 25 months (range, 12-42 months). The type of femoral stem used was cemented in 7 cases ( 4 in primary THA and 3 in revision THA) and uncemented in 30 cases ( 26 in primary THA

Table 1. Baseline Characteristics of Patients

\begin{tabular}{lc}
\hline \hline Characteristic & Data \\
\hline Total fracture (n) & 37 \\
Age $(\mathrm{yr})$ & $75(43-90)$ \\
Gender, male/female & $16 / 21$ \\
Interval of fracture & $7 \mathrm{yr} 3 \mathrm{mo}(15 \mathrm{~d}-30 \mathrm{yr})$ \\
Traumatic mechanism of fracture & \\
$\quad$ Slip down & 33 \\
$\quad$ Minor traffic accident & 1 \\
Non-traumatic mechanism of fracture & \\
$\quad$ Intraoperative fracture & 2 \\
$\quad$ During hip joint movement & 1 \\
Cementless stem/cemented stem & $30 / 7$ \\
\hline
\end{tabular}

Values are presented as number only or median (range).

Table 2. New Principle of Surgical Treatment for Periprosthetic Femoral Fracture

\begin{tabular}{lc}
\hline \hline Vancouver type & Method \\
\hline A & Open reduction with wiring \\
B1 & Open reduction with double plate fixation \\
B1-nonunion & Revisional total hip arthroplasty with long revision stem \\
B2 & Revisional total hip arthroplasty with long revision stem \\
B3 & Revisional total hip arthroplasty with long revision stem \\
C & Open reduction with double plate fixation \\
\hline
\end{tabular}

B1-nonunion: defined as nonunion fracture after surgical treatment of Vancouver B1 type. 
and 4 in revision THA). The causes of fractures, a history of femoral head fracture and characteristics were analyzed using medical records. Local assumed risk factors for fractures (e.g., bone defect or stem loosening), were evaluated using pre- and post-fracture radiographs. As seen in Table 1, fractures were caused by a fall or minor trauma $(n=34)$ or non-traumatic event $(n=3)$.

Femoral stem type usage by Vancouver classification is as follows: 8 type $\mathrm{A}$ ( 7 uncemented and 1 cemented), 13 type B1 (12 uncemented and 1 cemented stems), 2 type B2 (2 uncemented stems), 2 type B3 (1 uncemented and 1 cemented stems) and 9 type $C$ ( 6 uncemented and 3 cemented stems). Although not described in the Vancouver classification, we included 3 cases experiencing nonunion without stem loosening after surgical management of Vancouver type B1 fractures (hereinafter referred to as "B1-nonunion"). All operations were performed by a single surgeon. Advanced hip arthroscopy surgical approaches varied depending on the fracture type: i) open reduction with wiring for Vancouver type A, ii) open reduction with double-plate fixation for Vancouver type B1 whether using cemented or uncemented stems in primary THA, iii) revision THA with a long stem for Vancouver type B1-nonunion B2 and B3, and iv) open reduction with double-plate fixation for Vancouver type $\mathrm{C}$ fractures (Table 2).

Clinical results were evaluated using the Beals and Tower's criteria (e.g., implant stability, fracture healing and onset of complications) until fracture union throughout the 10-month follow-up period) $)^{33}$ and are presented in Table 3.

Fracture union was defined radiologically (anteroposterior and lateral) as the time of callus formation and capacity for weight-bearing without pain. Stem stability was determined based on the appearance of radiolucent lines around the stem, and subsidence and loosening were examined ${ }^{10)}$. Bone mineral density (BMD) was examined in 27 of 37 cases within the first 6 weeks following surgery. Dual-energy x-ray absorptiometry (LUNAR BX-1L; GE Medical Systems, WI, Madison, USA) was used to determine BMD at the femoral neck and trochanter. The T-score was used to compare BMD with the maximum BMD of the healthy population.
Osteoporosis was defined based on the WHO criteria ${ }^{11}$. Statistical analyses were performed with SPSS version 17.0 (SPSS Inc., Chicago, IL, USA). The chi-square and Fisher's exact tests were used to test the correlation between osteoporosis diagnosed based on BMD T-scores of less than -2.5 and radiological findings assessed with Beals and Tower's criteria. $P$-values were considered significant at $P<0.05$.

\section{RESULTS}

Using Beals and Tower's criteria, radiological results were deemed excellent in $33(89.2 \%)$ and poor in $4(10.8 \%)$ of 37 patients. The risk factors for periprosthetic femoral fracture were a history of revision THA ( $\mathrm{n}=7$ cases; 3 treated with cemented stems and 4 with uncemented stems), a history of internal fixation using plates and screws $(\mathrm{n}=3)$, osteolysis $(n=2)$ and cortical defect $(n=2)$, and osteoporosis $(n=11)$. The average time to fracture healing 24 weeks (range, 17-39 weeks) after surgery. Revision THA was performed in 1 case with nonunion at 30 months postoperatively.

Clinical results were excellent in all 8 cases with Vancouver type A fractures and there were no instances of stem loosening, nonunion or osteolysis during mid- and longterm follow-up. All 13 cases with Vancouver type B1 fractures showed excellent outcomes, and no instances of metal failure or nonunion were detected at short-, midand long-term follow-up (Fig. 1). Two of 3 patients with type B1-nonunion fractures were referred to our hospital due to nonunion during a follow-up of more than 6 months after internal fixation using single plate for management of periprosthetic femoral fractures from other hospitals. These two patients underwent revision THA with a long stem and wire after removal of preexisting plates and showed excellent results during mid- and long-term follow-up. The other patient with a type B1-nonunion fracture showed nonunion at 5 months follow-up despite revision THA using a long stem, and underwent another revision THA with the use of a longer stem with a larger diameter, wire and cortical allograft. Fracture union was achieved without

Table 3. Beals and Tower's criteria

\begin{tabular}{lccc}
\hline \hline Outcome & Arthroplasty & & Fracture \\
\hline $\begin{array}{l}\text { Excellent } \\
\text { Good }\end{array}$ & Stable & and & Healed with minimal deformity without shortening \\
& Stable & or & Healed with moderate deformity and shortening \\
Poor & $\begin{array}{c}\text { Subcidence } \\
\text { Loose }\end{array}$ & or & Nonunion, sepsis, or new fracture with severe deformity and shortening \\
\hline
\end{tabular}




\section{Hip \& Pelvis}

Joong-Myung Lee et al. Treatment of Periprosthetic Fracture Following Hip Arthroplasty

stem loosening at two-year follow-up in this patient (Fig. 2). Both patients with Vancouver type B2 fractures had excellent outcomes without stem subsidence or loosening at a mid- and long-term follow-up of more than 2 years. Of the 2 cases with a type B3 fracture, clinical results were excellent in 1 and poor in 1 . A patient achieved an excellent result without stem loosening 18 months after revision THA using a long stem for treatment of a fracture due to a fall after primary THA using a cemented stem. The other B3 fracture patient with a poor outcome had osteoporosis (T-score of -3.2 at the time of primary THA) and stem loosening 20 years after primary surgery with an uncemented stem, and then underwent revision THA using a long stem and wire due to a fracture after a fall. Since delayed union was observed at the 30-month followup, revision THA with a long stem was performed. A type
B1 fracture occurred 14 days after revision due to reinjury, and fracture union was obtained 12 months after double-plate fixation. Of the 9 cases with a Vancouver type $\mathrm{C}$ fracture, clinical results were excellent in 6 and poor in 3. No cases of nonunion or metal failure were observed in the 6 excellent cases (Fig. 3). Of the 3 cases with poor results, a patient with osteoporosis (T-score of -3.2) underwent revision surgery using double plates long enough to cover the full length of the femur due to a new fracture at the distal plate of the femoral shaft at the 1-month follow-up after fixation using double plates and cables, and achieved excellent results at the 18-month follow-up. Another patient with a poor outcome in the treatment of a Vancouver type $\mathrm{C}$ fracture received internal fixation with plates due to nonunion and metal failure observed at the 2-month followup after double-plate fixation; excellent results were observed
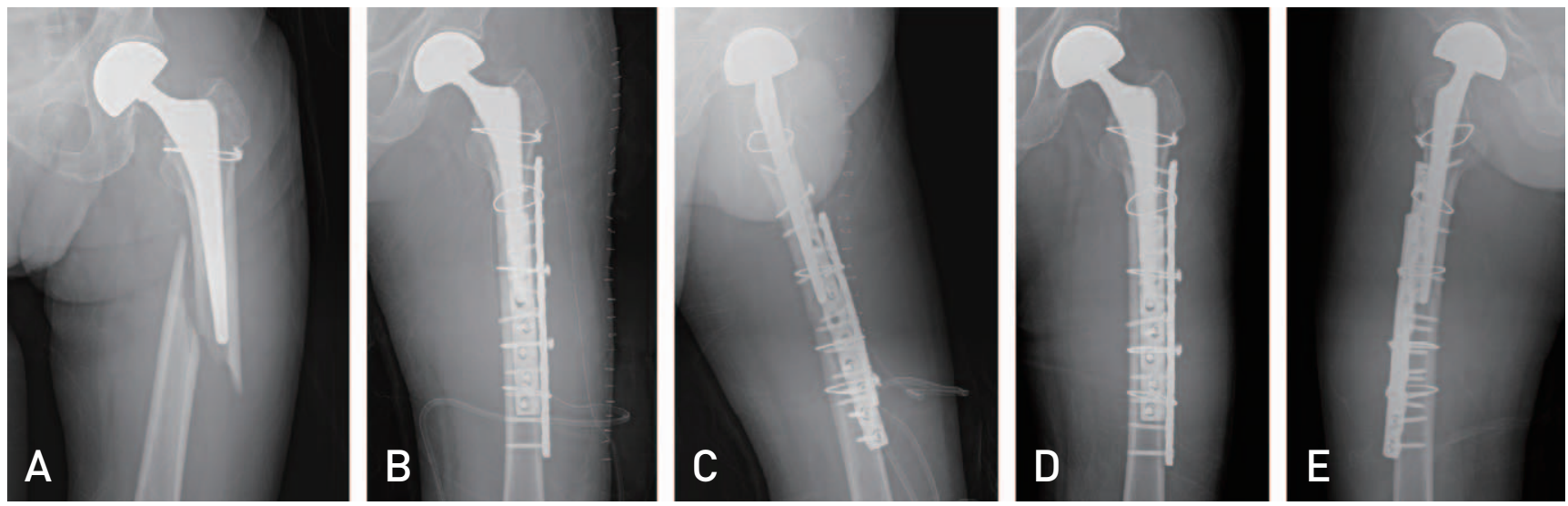

Fig. 1. (A) In a 84-year-old male patient, a Vancouver type B1 fracture occurred when he slipped down. (B, C) Open reduction and internal fixation was performed with double plates and cables. (D, E) At 12 months after operation, X-ray shows bony union and no evidence of femoral stem loosening.
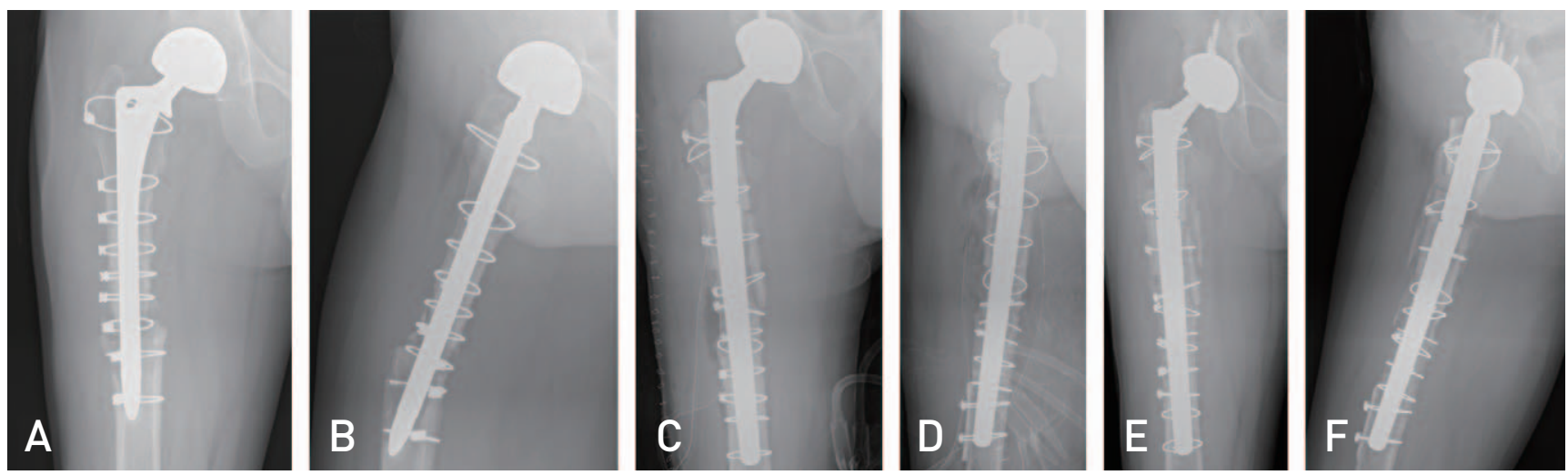

Fig. 2. In a 54-year-old male patient, a Vancouver type B1 fracture occurred when he slipped down. Revision hip arthroplasty was then performed at another hospital. X-rays taken 5 months after the revision hip arthroplasty shows nonunion; (A) anteroposterior view, (B) lateral view. (C, D) Cup and stem revision was performed using a longer revision femoral stem. (E, F) An X-ray taken 24 months after revision total hip arthroplasty shows bony union and stable stem fixation. 


\section{Hip \& Pelvis}

Hip Pelvis 30(2): 78-85, 2018

24 months after revision surgery. The other patient with a type $\mathrm{C}$ fracture associated with osteoporosis (T-score of -5.4) underwent blade-plate internal fixation due to a new fracture occurred at the distal portion of the femoral stem 2 weeks after insertion of bone cement and internal fixation using double plates and cables; excellent outcomes were observed 24 months after revision surgery (Fig. 4, Table 4).
BMD T-scores were assessed in 27 out of 37 patients and the mean was -2.6 . Of these 27 patients, the T-score breakdowns were: i) normal ( -2.0 or above) in 10 , ii) osteopenia ( -2.0 to -2.5$)$ in 6 , and iii) osteoporosis $(-2.5$ or below). Of interest, the average T-score was -3.6 in the 9 patients with type $\mathrm{C}$ fractures and all had osteopenia or osteoporosis. According to radiological results evaluated with Beals and Tower's criteria, the mean T-score was
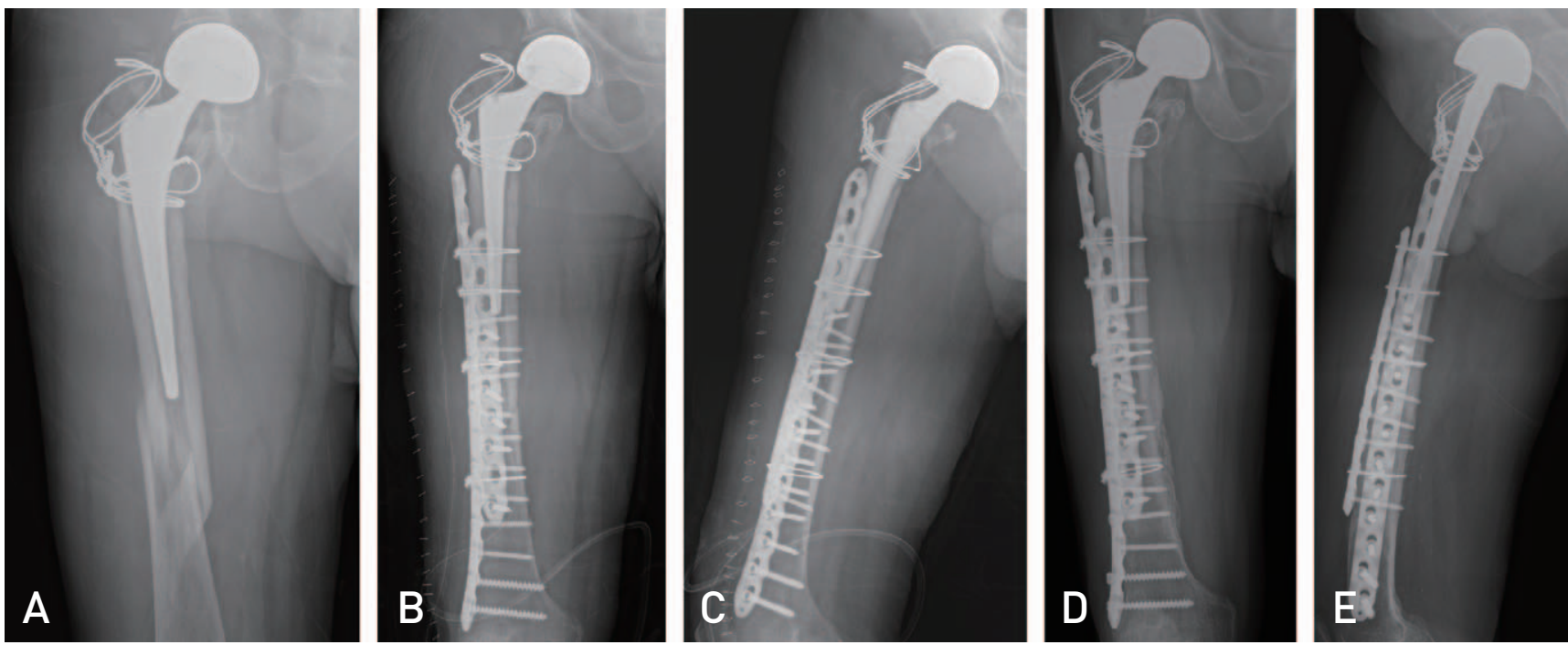

Fig. 3. (A, B) In an 82-year-old male patient, a Vancouver type C fracture occurred when he slipped down. (C, D) Open reduction and internal fixation was performed with double plates lone plate is a long plate that include the femur total length) and cables. (E) X-rays taken 12 months after operation show bony union and no evidence of newly fracture around femoral stem.
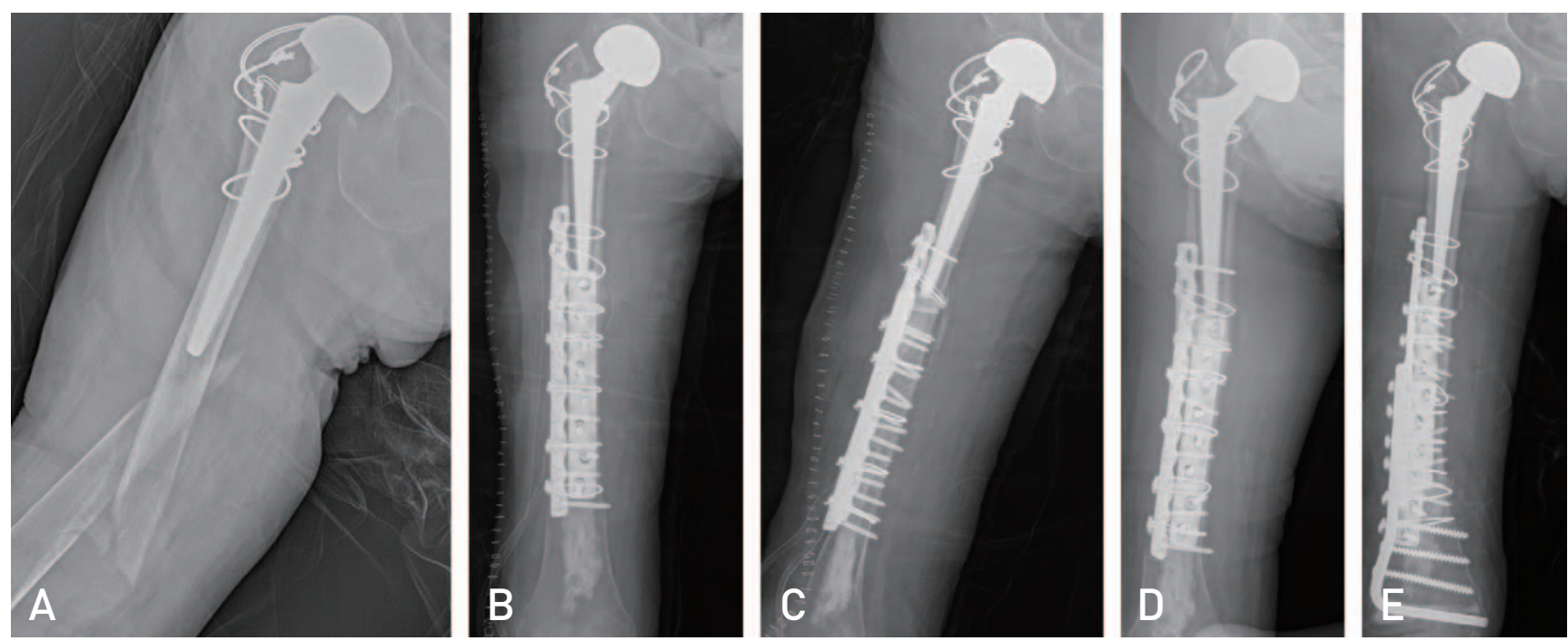

Fig. 4. (A) In an 80-year-old female patient, a Vancouver type C fracture occurred when she slipped down. (B, C) Open reduction and internal fixation was performed with double plates and cables. (D) Two weeks after the initial operation, a new fracture occurred at the distal femoral stem site. (E) An X-ray taken 24 months after the revision surgery shows bony union and femoral stem stability. 
-4.4 in those with a poor prognosis $(n=4)$, and -2.3 in those with a good prognosis $(n=23)$. Osteoporosis was statistically correlated to patients characterized as poor using the Beals and Tower's criteria $(P<0.05)$ (Table 5).

\section{DISSCUSSION}

Risk factors for periprosthetic femoral fracture are relate to weak bone quality, systemic factors (e.g., old age and osteoporosis) and local factors (e.g., osteolysis and stem loosening $)^{12}$. In this study, of the 37 patients, $40.7 \%$ had osteoporosis and $22.2 \%$ had osteopenia, and $18.9 \%$ had a history of revision surgery due to aseptic loosening and other causes. The leading cause of fractures was minor trauma $(91.9 \%)$. Based on these findings, we have inferred that mild traumatic injury (e.g., a fall) can induce a fracture in patients experiencing bone weakness. In the past, stem loosening was the most common risk factor for periprosthetic femoral fractures with the use of cemented stems in THA. However, a recent trend toward increased use of uncemented stems has decreased the rate of stem loosening, and instead the rate of systemic diseases (e.g., osteoporosis) has risen along with the increased incidence of periprosthetic femoral fracture with age ${ }^{7}$. In the authors' previous study, $28 \%$ of patients had stem loosening prior to periprosthetic femoral fracture, but here, stem loosening was reduced to $14 \%$ while the incidence of osteoporosis increased to $40.7 \%$. With recent advances in the stability of uncemented stems, the use of uncemented stem fixation has increased leading to more frequent periprosthetic femoral fractures. Compared to the authors' previous study, the use of cemented stem has dramatically declined from $50 \%$ to $19 \%$.

The treatment goals of any periprosthetic femoral fracture include, but are not limited to, properly aligned union, stem stability, recovery of pre-fracture functional mobility, and early mobilization ${ }^{5}$. The incidences of nonunion and stem loosening are high due to osteoporosis, absorption of the femoral head, cortical defect, local bone defect of the femoral head and others. The rate of refracture after internal fixation is high due to stress concentration factors (e.g., screw holes, plate ends, and local bone defects of the femoral head $)^{6}$.

The standard treatment for type B1 fractures are accurate reduction and rigid internal fixation, but internal fixation can be challenging in older patients with weakened bone quality due to absorption of the femoral head, cortical defect and local bone defect of the femoral head.

Moreta et al. ${ }^{10}$ obtained excellent or good clinical outcomes in $21(87.5 \%)$ of 24 cases with Vancouver type B1 fractures and poor results in the other 3 cases after performing open reduction and internal fixation (ORIF) using a plate and cerclage wires. In these authors' previous study, satisfactory results were achieved in 17 of $20(85.0 \%)$ cases with type B1 fractures using cerclage wiring alone or plate internal fixation combined with cerclage wiring ${ }^{6}$. The current study

Table 4. Outcomes of Periprosthetic Fracture Following to the Vancouver Classification

\begin{tabular}{lccc}
\hline \hline Type & Total $(\mathrm{n})$ & Beals and Tower's Criteria $(\mathrm{n})$ & Complication \\
\hline A & 8 & Excellent: 8 & \\
B1 & 13 & Excellent: 13 & \\
B1-nonunion & 3 & Excellent: 3 & \\
B2 & 2 & Excellent: 2 & Nonunion \& \\
B3 & 2 & Excellent: 1 & New fracture occur distal implant site \\
& & Poor: 1 & New fracture occur distal plate site \\
C & 9 & Excellent: 6 & \\
& & Poor: 3 & \\
\hline
\end{tabular}

Table 5. Distribution of the Osteoporosis and Surgical Treatment Outcome

\begin{tabular}{|c|c|c|c|c|}
\hline \multirow{2}{*}{ T-score } & \multicolumn{2}{|c|}{ Beals and Tower's criteria } & \multirow{2}{*}{ Total } & \multirow{2}{*}{$x^{2}(P$-value $)$} \\
\hline & Excellent & Poor & & \\
\hline$z-2.5$ & $16(100.0)$ & $0(0.0)$ & 16 & \multirow{2}{*}{$6.830 *(0.019)$} \\
\hline$<-2.5$ & $7(63.6)$ & $4(36.4)$ & 11 & \\
\hline
\end{tabular}

Values are presented as number $(\%)$.

$* P<0.05$. 
applied new treatment principles to 13 cases with Vancouver type B1 and excellent results were achieved in all 13 cases $(100 \%)$ after internal fixation using double plates and ORIF using cables in the lateral and anterior parts of the femoral head. Better surgical outcomes appear to be attributable to the greater number of screws used in double-plate fixation and greater stability with rigid fixation of the lateral and anterior parts of the femoral head in older patients with poor bone quality. In a biomechanics study, Wilson et al. ${ }^{13}$ concluded that an insignificant difference was found in bone strength and stability between double-plate fixation and fixation with a plate combined with cortical bone allograft.

When nonunion occurs after surgical treatment of type B1 fractures, revision surgery is done after primary fixation with plates and cables. In this procedure, previously implanted screws and plates should be removed which increases the risk of reduced fracture healing as bone quality becomes poor at the screw insertion sites and the nonunion fracture site. Additionally, decreased femoral head blood flow is associated with injuries to the soft tissue and periosteum from the previous surgery. A revision ORIF using plates is expected to increase the risk of nonunion and stem loosening. Thus, revision THA for Vancouver type B1 fractures is thought to be more helpful in preventing complications such as stem loosening, nonunion or refracture and providing postoperative stability of the prosthetic components. Although type B1 fractures of cemented stems are classified radiographically as type B1 fractures, this fracture is more likely to experience stem loosening during surgery and should be treated with revision THA by classifying it as a type B2 fracture. Therefore, the stability of the stem should be confirmed when using cemented stems.

A variety of revision stems are used to manage B2 and B3 factures, but distal fixation using extensively porouscoated stems is preferred and several authors have achieved successful results ${ }^{14}$. Since type B3 fractures have severe bone loss in the proximal femur due to osteoporosis, osteolysis and compound fracture, surgical treatment can be more challenging in the periprosthetic fracture group. Based on surgeon's experience and fracture pattern, rigid fixation of the stem should be carried out using bone cement, autogenous graft and impaction allograft.

The standard treatment for type C fractures is ORIF. Moreta et al. ${ }^{10)}$ documented excellent or good clinical results in 3 out of 5 cases $(60 \%)$ and poor results in the remaining 2 cases of type $C$ fractures using ORIF. In these authors' previous study, metal failure occurred in 1 of 2 cases with type $\mathrm{C}$ fractures and this patient was treated with doubleplate fixation and autologous bone grafting ${ }^{6}$. Type $\mathrm{C}$ fractures are significantly associated with osteoporosis. In this study, when BMD was assessed, 7 of 9 patients with type $\mathrm{C}$ fractures had osteopenia or osteoporosis (T-score range, -2.1 to -5.4 ). Based on these authors' experiences, efforts should be undertaken to achieve rigid fixation with the overlap of the proximal plate and the stem by using a plate long enough to cover the full length of the femur and insertion of cortical screws as many as possible particularly in patients with type $\mathrm{C}$ fractures associated with osteoporosis due to weak bones, in order to minimize the risk of postoperative refracture.

\section{CONCLUSION}

Here we see that applying new hip THA treatment principles led to superior results when compared with the results of these authors' previous study. The following surgical approaches according to Vancouver types are thought to be helpful: i) internal fixation using double plates (instead of a single plate) for a type B1 fracture, ii) revision THA for a type B1 nonunion, and iii) internal fixation using a longer plate in combination of insertion of bone cement, if necessary, for a type $\mathrm{C}$ fracture associated with osteoporosis. For the treatment of periprosthetic femoral fractures following THA, appropriate surgical intervention should be chosen according to the Vancouver classification. In addition, osteoporosis is considered to be a major prognostic factor for the outcomes of surgical treatment.

\section{CONFLICT OF INTEREST}

The authors declare that there is no potential conflict of interest relevant to this article.

\section{REFERENCES}

1.Jämsen E, Salonen H, Taukojärvi A, et al. P-436: The incidence of periprosthetic femoral fractures after total hip replacement in the Pirkanmaa Hospital District, Finland during 2002-2010. Eur Geriatr Med. 2015;6 Suppl 1:S149.

2. Richards CJ, Garbuz DS, Masri BA, Duncan CP. Vancouver type B3 periprosthetic fractures: evaluation and treatment. Instr Course Lect. 2009;58:177-81.

3. Beals RK, Tower SS. Periprosthetic fractures of the femur. An analysis of 93 fractures. Clin Orthop Relat Res. 1996;(327): 238-46.

4. Lindahl H, Malchau H, Herberts P, Garellick G. Periprosthetic femoral fractures classification and demographics of 1049 


\section{Hip \& Pelvis}

Joong-Myung Lee et al. Treatment of Periprosthetic Fracture Following Hip Arthroplasty

periprosthetic femoral fractures from the Swedish National Hip Arthroplasty Register. J Arthroplasty. 2005;20:857-65.

5. Lee JM. Periprosthetic femoral fractures after hip arthroplasty. J Korean Hip Soc. 2011;23:174-83.

6. Lee JM, Hwang DW, Choi HJ, Seol YD, Lee DS. Treatment of periprosthetic femoral fractures after hip arthroplasty. $J$ Korean Orthop Assoc. 2010;45:114-9.

7.Lindahl H. Epidemiology of periprosthetic femur fracture around a total hip arthroplasty. Injury. 2007;38:651-4.

8. Duncan CP, Masri BA. Fractures of the femur after hip replacement. Instr Course Lect. 1995;44:293-304.

9. Garbuz DS, Masri BA, Duncan CP. Periprosthetic fractures of the femur: principles of prevention and management. Instr Course Lect. 1998;47:237-42.

10. Moreta J, Aguirre U, de Ugarte OS, Jáuregui I, Mozos JL. Functional and radiological outcome of periprosthetic femoral fractures after hip arthroplasty. Injury. 2015;46:292-8.

11. Hannan MT, Felson DT, Anderson JJ. Bone mineral density in elderly men and women: results from the Framingham osteoporosis study. J Bone Miner Res. 1992;7:547-53.

12. Yoon BH, Lee YK, Jo WL, Ha YC, Choi DH, Koo KH. Incidence and risk period of periprosthetic femoral fracture after cementless bipolar hemiarthroplasty in elderly patients. J Arthroplasty. 2016;31:1326-30.

13. Wilson D, Frei H, Masri BA, Oxland TR, Duncan CP. A biomechanical study comparing cortical onlay allograft struts and plates in the treatment of periprosthetic femoral fractures. Clin Biomech (Bristol, Avon). 2005;20:70-6.

14. Kim Y, Tanaka C, Tada H, Kanoe H, Shirai T. Treatment of periprosthetic femoral fractures after femoral revision using a long stem. BMC Musculoskelet Disord. 2015;16: 113. 